\title{
Téoros
}

Revue de recherche en tourisme

\section{Les comportements de vacances des jeunes des États-Unis et du Canada}

\section{Jean Stafford}

Volume 20, numéro 3, automne 2001

Comportements touristiques

URI : https://id.erudit.org/iderudit/1071626ar

DOI : https://doi.org/10.7202/1071626ar

Aller au sommaire du numéro

Éditeur(s)

Université du Québec à Montréal

ISSN

0712-8657 (imprimé)

1923-2705 (numérique)

Découvrir la revue

Citer cet article

Stafford, J. (2001). Les comportements de vacances des jeunes des États-Unis et du Canada. Téoros, 20(3), 5-10. https://doi.org/10.7202/1071626ar d'utilisation que vous pouvez consulter en ligne.

https://apropos.erudit.org/fr/usagers/politique-dutilisation/ 


\section{Les comportements de vacances des jeunes des États-Unis et du Canada}

\section{Jean Stafford}

e présent article porte principalement sur les vacances' des jeunes des ÉtatsUnis et du Canada ; il traite aussi des loisirs de ces deux groupes de jeunes. Cette analyse n'est qu'une partie d'un rapport de recherche effectué sous la coordination du Bureau international du tourisme social section des Amériques (BITS-Amériques) pour le compte de la Commission canadienne du tourisme, avec la collaboration scientifique du Centre international de formation et de recherche en tourisme (CIFORT) de l'École des sciences de la gestion de l'Université du Québec à Montréal.

\section{La méthodologie ef la population étudiée}

L'enquête sur les activités et les préférences des jeunes en matière de voyages a été réalisée pour le compte de la Commission canadienne du tourisme et de différents ministères fédéraux et provinciaux. La collecte des données (les entrevues) s"est faite du 27 septembre 1999 au 16 avril 2000. L'enquête comprenait un sondage téléphonique et un sondage postal effectués aux États-Unis et au Canada.

Le sondage téléphonique s'est fait auprès de 28397 répondants aux États-Unis et de 18385 au Canada : il s'agissait de deux sondages stratifiés et aléatoires. Quant au sondage postal, le nombre des répondants était de 6405 aux Êtats-Unis et de 5405 au Canada. La firme Price Coopers Waterhouse était responsable de la collecte des données aux États-Unis, tandis que
Statistique Canada avait ce mandat au Canada. La marge d'erreur théorique sur les réponses à $95 \%$ (19 fois sur 20 ) est d'environ $2 \%$ pour les deux sondages téléphoniques et d'environ $5 \%$ pour les sondages postaux.

Pour les fins de cette étude sur les comportements touristiques des jeunes des États-Unis et du Canada, nous avons extrait le groupe des dix-huit - vingt-quatre ans de l'échantillon original. Le traitement et l'interprétation des données porteront uniquement sur ce groupe d'âge ; ce découpage correspond à celui effectué par différentes études internationales ; il permet de comparer les comportements touristiques des jeunes de differents pays.

\section{Les départs en vacances, la durée ef les destinations}

Le tableau 1 présente le nombre de semaines de vacances auxquelles le répondant a droit en 1999. Nous y remarquons que les jeunes Canadiens ont droit à moins de semaines de vacances que les jeunes américains : $38 \%$ des jeunes Canadiens n'ont droit à aucune semaine de vacances comparativement à $28 \%$ des jeunes Américains. Malgré cela, la moyenne des semaines de vacances est identique dans les deux groupes $(1,9$ semaine de vacances).

Le tableau 2 illustre le nombre de jours consacrés aux voyages d'agrément en 1999. Nous pouvons constater que les différences sont minimes entre les deux groupes de jeunes: la moyenne du nombre de jours de vacances est de 11,3 jours pour les jeunes Canadiens et de 10 jours pour les jeunes Américains. Les jeunes Canadiens sont plus nombreux ( $45 \%$ ) que les jeunes Américains $(39 \%)$ dans la catégorie huit jours et plus.

Le tableau 3 montre les destinations de voyages des deux dernières années. Les jeunes Américains sont $12 \%$ à visiter le Canada allors que 31 \% des jeunes Canadiens visitent les États-Unis. Nous pouvons constater que l'échange touristique

\begin{tabular}{|l|c|c|}
\hline & Américains & Canadiens \\
\hline LE NOMBRE DE SEMAINES & $\%$ & $\%$ \\
\hline Aucune & 22 & 38 \\
1 semaine de vacances & 22 & 9 \\
2 semaines de vacances & 30 & 30 \\
3 semaines de vacances & 8 & 12 \\
4 semaines de vacances & 7 & 7 \\
Plus de 4 semaines & 100 & 100 \\
\hline TOTAL & A & \\
\hline
\end{tabular}


7anisu:

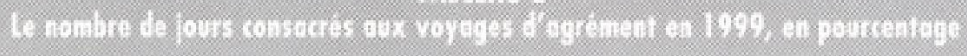

\begin{tabular}{|l|c|c|}
\hline LE NOMBRE DE JOURS & Américains & Canadiens \\
\hline Aucun & $\%$ & $\%$ \\
\hline De 1 à 7 jours & 26 & 26 \\
De 8 à 14 jours & 35 & 29 \\
De 15 à 21 jours & 18 & 23 \\
Plus de 22 jours & 10 & 12 \\
\hline TOTAL & 100 & 100 \\
\hline
\end{tabular}

\begin{tabular}{|c|c|c|}
\hline \multicolumn{3}{|c|}{ 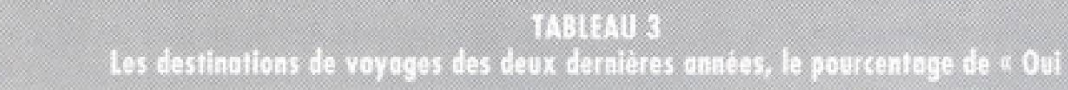 } \\
\hline LES DESTINATIONS & $\begin{array}{c}\text { Américains } \\
\qquad \%\end{array}$ & $\begin{array}{c}\text { Canadiens } \\
\%\end{array}$ \\
\hline Etats-Unis & 71 & 31 \\
\hline Canada & 12 & 72 \\
\hline Mexique/Caraibes & 17 & 6 \\
\hline Autres pays & 12 & 6 \\
\hline
\end{tabular}

\begin{tabular}{|c|c|c|}
\hline \multicolumn{3}{|c|}{ 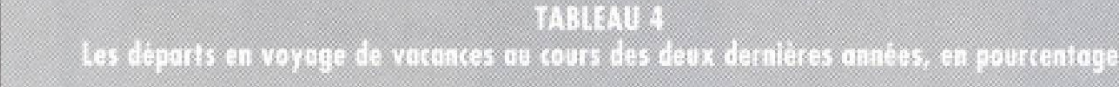 } \\
\hline LES DÉPARTS EN VACANCES & Américains & Canadiens \\
\hline & $\%$ & $\%$ \\
\hline Oui & 82 & 72 \\
\hline Non & 18 & 28 \\
\hline Total & 100 & 100 \\
\hline
\end{tabular}

\begin{tabular}{|c|c|c|}
\hline \multicolumn{3}{|c|}{ 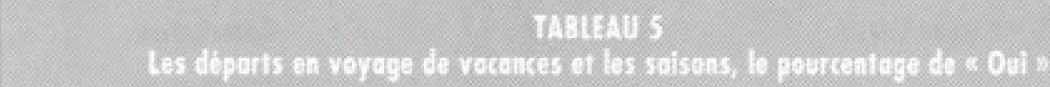 } \\
\hline LES SAISONS & Américains & Canadiens \\
\hline & $\%$ & $\%$ \\
\hline En hiver & 49 & 47 \\
\hline Au printemps & 48 & 35 \\
\hline En été & 78 & 77 \\
\hline En automne & 37 & 24 \\
\hline
\end{tabular}

est très inégal. En effet, l'attrait des ÉtatsUnis est plus ellevé chez les Canadiens que l'attrait du Canada pour les Américains ; les jeunes Américains voyagent plus à l'étranger que les jeunes canadiens et l'attrait du Mexique et des îles des Caraïbes est plus élevé chez les jeunes Américains que l'attrait du Canadal.

Le tableau 4 illustre les départs en voyage de vacances des jeunes des deux pays ; les jeunes Américains sont un peu plus nombreux $(82 \%)$ que les jeunes Canadiens $(72 \%)$ à prendre des vacances.

La grande majorité des jeunes Américains et des jeunes Canadiens (tableau 5) prennent leurs vacances en été ( $78 \%$ des Américains et $77 \%$ des Canadiens). En hiver, les pourcentages sont à peu près identiques pour les deux groupes de jeunes (49\% des Américains et $47 \%$ des Canadiens). Les jeunes Américains sont plus nombreux à prendre des vacances au printemps ( $48 \%$ des Américains et $35 \%$ des Canadiens); la même situation se vit également à l'automne (37 \% des Améri= cains et $24 \%$ des Canadiens).

\section{Les formes \\ d'accompagnement, les voyages organisés et les forfaits}

Le quart des jeunes répondants voyage en solitaire $(27 \%$ des jeunes Américains et $25 \%$ des jeunes Canadiens). Environ $30 \%$ des jeunes répondants (tableau 6) ont fait au moins un voyage de vacances avec des amis ou des proches parents.

Les jeunes Américains sont un peu plus nombreux que les jeunes Canadiens (tableau 7) à prendre des vacances dans le cadre d'un voyage organise ( $21 \%$ des Américains et $14 \%$ des Canadiens). La très grande majorité des jeunes préferent organiser eux-mêmes leurs vacances.

En ce qui concerne l"utilisation de forfaits (tableau 8), les jeunes Américains sont un peu plus nombreux que les jeunes Canadiens à profiter de ce mode de voyage : il faut souligner que la très grande majorité des jeunes utilisent rarement ou jamais des forfaits pour les vacances. 


\section{L'hébergement en vacances et les sources $d^{\prime}$ 'information utilisées dans la planification des voyages de vacances}

Pour les Américains et les Canadiens, les types d'hébergement les plus utilisés en vacances (tableau 9) sont :

- maison/appartement d’amis ou de proches parents ;

- hôtel/centre de villégiature/auberge rurale ;

- motel.

Pour les autres modes d'hébergement, les pourcentages des jeunes utilisateurs sont beancoup plus faibles.

Les sources d'information utilisées lors de la planification des voyages de vacances, dans les deux dernières années, apparaissent au tableau 10. Pour les jeunes Américains, les sources d'information qui ont un pourcentage supérieur à $40 \%$ sont :

- conseils d'amis ou de proches $(55 \%)$;

- expériences antérieures $(52 \%)$ :

- Internet $(47 \%)$.

Pour les jeunes Canadiens, il n'y a que deux sources d'information qui dépassent $40 \%$ :

- conseils damis ou de proches $(60 \%)$;

- expériences antérieures $(56 \%)$.

Les conseils des amis et de proches ainsi que les expériences passées sont les sources les plus importantes d'information touristique pour les jeunes des deux pays. Il faut noter les différences marquées des pourcentages entre les jeunes Américains et les jeunes Canadiens en ce qui concerne l'utilisation de l'Internet: en effet, les jeunes Américains sont deux fois plus nombreux que les jeunes Canadiens à utiliser 1'Internet quand ils planifient un voyage.

\section{Les comportements touristi- ques des jeunes Américains et des jeunes Canadiens}

Les jeunes des deux groupes prennent, en moyenne, deux semaines de vacances annuellement. Ces vacances durent, en moyenne, onze jours pour les jeunes ca-

\begin{tabular}{|c|c|c|}
\hline \multicolumn{3}{|c|}{ 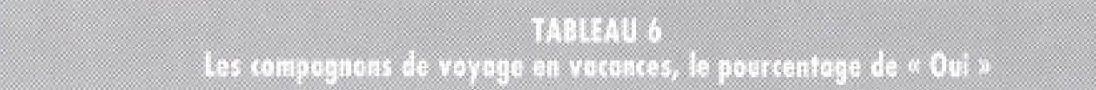 } \\
\hline L'ACCOMPAGNEMENT & Américains & $\begin{array}{c}\text { Canadiens } \\
\%\end{array}$ \\
\hline - Seul, non accompagné & 27 & 25 \\
\hline $\begin{array}{l}\text { - Avec des amis ou des associés } \\
\text { (sans membre de votre famille) }\end{array}$ & 40 & 40 \\
\hline $\begin{array}{l}\text { - Avec lé/la conjointleV/partenaire. } \\
\text { en couple (sans enfant) }\end{array}$ & 36 & 37 \\
\hline $\begin{array}{l}\text { - Avec la famille immédiate, } \\
\text { y compris des enfants }\end{array}$ & 43 & 32 \\
\hline $\begin{array}{l}\text { - Avec la famille immédiate et des amis } \\
\text { ou d'autres proches }\end{array}$ & 37 & 31 \\
\hline - Autres & 9 & 7 \\
\hline
\end{tabular}

\begin{tabular}{|c|c|c|}
\hline \multicolumn{3}{|c|}{ 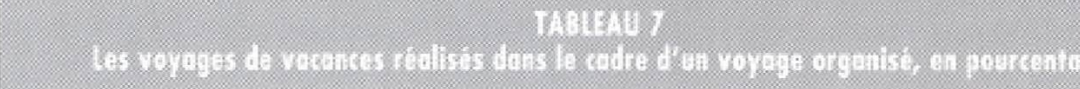 } \\
\hline UN VOYAGE ORGANISE & Américains & $\begin{array}{c}\text { Canadiens } \\
\%\end{array}$ \\
\hline Oui & 21 & 14 \\
\hline Nan & 79 & 86 \\
\hline Total & 100 & 100 \\
\hline
\end{tabular}

\begin{tabular}{|c|c|c|}
\hline \multicolumn{3}{|c|}{ 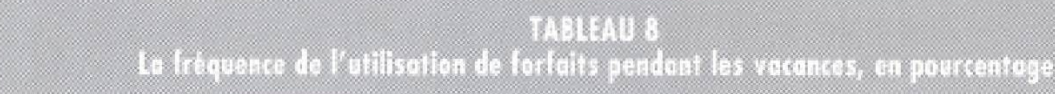 } \\
\hline L'UTILISATION DE FORFAITS & $\begin{array}{c}\text { Américains } \\
\%\end{array}$ & $\begin{array}{c}\text { Canadiens } \\
\%\end{array}$ \\
\hline Toujours & 6 & 4 \\
\hline Parfois & 22 & 21 \\
\hline Rarement & 27 & 19 \\
\hline Jamais & 43 & 49 \\
\hline Ne s'applique pas & 2 & 7 \\
\hline Total & 100 & 100 \\
\hline
\end{tabular}

nadiens et dix jours pour les jeunes américains.

La majorité des jeunes répondants voyagent dans leut propre pays. La proportion de voyages de vacances à l'étranger est assez faible et la plus grande partie de ces vacances se passent aux États-Unis, au Canada ou au Mexique.

La plupart des jeunes vont en vacances surtout en été (plus de $75 \%$ ) ; les autres saisons sont aussi représentées, mais dans des pourcentages moindres (environ $45 \%$ ). Les jeunes vont en vacances avee des amis ou des parents et près de $25 \%$ voyagent seuls.

Les jeunes sont peu nombreux à utiliser les forfaits et à voyager dans le cadre d'un voyage organisé. Les modes d'hébergement les plus utilisés en vacances sont

- maison ou appartement d'amis ou de proches parents ;

- hôtel, centre de villégiature ou auberge rurale.

Pour préparer leurs voyages de vacances, les jeunes s'appuient surtout sur les conseils d'amis ou de proches parents, sur 


\section{Panorama des vacances des jeunes Français de 15 à 24 ans}

\section{Martine Geronimi}

En France, deux grandes enquêtes sont à la base des données les plus récentes sur le tourisme des jeunes de 15 a 24 ans. En novembre 1998, l'Observatoire National du Tourisme publiait, dans la collection Les Essentiels du tourisme, un fascicule intitule Le Tourisme des 15 à 24 ans en France. Cette étude faisait état des vacances des jeunes Français, mais aussi des jeunes étrangers du même agge qui visitent la France. Deux ans plus tôt, l'Union Nationale des Associations de Tourisme (UNAT) colligeait une enquête menée auprès de 1200 jeunes âgés de 14 à 25 ans. Le but du présent article est de dresser un portrait qualitatif des vacances des jeunes Français. En effet, les variables des enquêtes françaises étant différentes de celles retenues pourl'enquête sur les vacances des jeunes du Canada et des États-Unis, il s'avérait difficile, voire incohérent, de faire une étude comparative. Nous voulons répondre à quelques questions simples afin de mettre en lumière les spécificités de ce panorama du tourisme des jeunes Français.

\section{Combien sont-ils ?}

Au premier janvier 2000 , la population française était estimée à 59325600 personnes, dont 7,7 millions de jeunes de 15 à 24 ans, soit environ 13 \% de la population. Cette classe d'âge est globalement en baisse du fait de la chute de la natalité et du vieillissement structurel de la population française. L'analyse des données permet de répartir la population des jeunes en deux types : scolarisés et actifs. Il faut également la scinder en deux tranches d'âge reflétant des variations psychologiques entre les jeunes; on distingue donc les jeunes de 15 à 19 ans et ceux de 20 à 24 ans. Les premiers sont scolarisés à $92 \%$ ( $75 \%$ en 1975), alors que les seconds ne sont scolarisés qu'à $46 \%$ ( $16 \%$ en 1975 ). On constate que les 20 à 24 ans sont dits actifs à $50 \%$, sachant que cette proportion inclut un grand nombre de chômeurs. Dans la décennie 1990 , le chômage des jeunes Français était fort élevé (en moyenne $25 \%$ ), du fait de la crise économique. En 2000 , ce chômage avoisine les $20 \%$. Il faut prendre en compte également le fait que ces jeunes occupent la plupart du temps un emploi à temps partiel. Cette clientèle ne dispose pas d'un gros revenu. Ce sont souvent des jeunes qui ont de la difficulté à vivre de façon autonome et qui consacrent peu d'argent à leurs vacances.

Il ressort plusieurs éléments de ces enquểtes :

- Premièrement, plus de deux millions de jeunes de 15 à 24 ans n' ont pas accès aux vacances. Ils sont, la plupart du temps, issus d'un milieu défavorisé et ils sont chômeurs ou tributaires d'un emploi précaire.

- Deuxièmement, le niveau socioculturel de la famille reste le critère premier dans les pratiques touristiques des jeunes de 15 à 24 ans.

- Troisièmement, la quasi-totalité du séjour des 15 à 24 ans est prise en charge par les parents, même si certains jeunes travaillent une partie de leurs vacances.

- Quatrièmement, les jeunes de 14 à 19 ans ont, depuis les années 1960, un taux de départ en vacances constamment supérieur à la moyenne française (en 2000 la moyenne française est de $70,8 \%$ contre $75 \%$ pour les jeunes de 14 a 19 ans). En revanche, depuis 1985, les jeunes de 20 à 24 ans ont un taux de départ en vacances inférieur à la moyenne française. Cette courbe descendante correspond à la montée du chômage pour cette classe d'âge, passant de $12,2 \%$ en 1980 à $28,5 \%$ en 1997.

\section{Quelles sont les pratiques culturelles les plus marquantes ?}

\section{Convivialité}

Cité par $89 \%$ des jeunes, le désir de convivialité met l'accent sur les sorties entre amis, sur le fait de partir en groupe, de visiter la famille, de faire des rencontres, mais également de prendre du repos au sein d'un groupe.

\section{Le désir de découverte}

Cette motivation vient en deuxième plan avec $75 \%$ des réponses. Ces pratiques englobent la recherche de dépaysement dans un site réputé reconnu pour sa beauté et pour son caractère exceptionnel.

\section{L'engagement personnel}

Les jeunes désirent vivre des expériences dans lesquelles ils peuvent s'engager personnellement. Cette réponse recueille $65 \%$ des préférences des jeunes. On y trouve le désir de se cultiver ( $27 \%$ ), d'apprendre une langue étrangère $(23 \%)$, de vivre une expérience religieuse ou philosophique $(2 \%)$ et, généralement, de vivre une activité de plein air $(45 \%)$. Le cam- 
ping caravaning est le type d'hébergement le plus utilisé par les jeunes.

\section{Quelles sont les destinations préférées des jeunes?}

La France reste de loin la destination touristique des jeunes Français avec 91 \% des séjours. Il est à noter que seuls $9 \%$ des séjours se réalisent à l'étranger avec une préférence marquée pour l'Europe dans $73 \%$ des cas. Le pays europén le plus visité est l'Espagne avec un quart des séjours européens, soit un peu plus de 90000 jeunes. L'Amérique (Canada et États-Unis) draine $9 \%$ des séjours à l'étranger, soit près de 45000 jeunes. Le choix des sites de destination en France fait ressortir trois caractéristiques :

1. On constate une nette baisse de la fréquentation de la campagne, réservée aux parents, au profit des séjours urbains, en particulier à l'automne. Le succès des parcs de loisirs français en témoigne avec, par exemple, plus de 3,5 millions de visiteurs à la Cité de la science de la Villette ou 3 millions de visiteurs au Futuroscope de Poitiers, sans compter les 12600000 visiteurs à Disneyland.

2. La montagne demeuré le lieu de séjour hivernal le plus recherché par les jeunes $(53,5 \%$ des sejours $)$ devant toutes les autres destinations (campagne $12 \%$, ville $13,5 \%$ ). Chez les adultes, la montagne ne représente que $33 \%$ des séjours hivernaux.

3. Le bord de mer attire toujours autant les jeunes en été ( $46 \%$ des sejours). Il faut remarquer que cette pratique estivale n'est pas spécifique aux jeunes, mais qu'elle est généralisée chez tous les Français.

\section{Conclusion}

Une typologie des styles de vie des jeunes Français en vacances nous est donnée par l'Observatoire National du Tourisme qui relève cinq types de comportements :

- Les « bronzés $*$ en vacances $(29,3 \%)$ font partie de la classe d'âge des 15 à 20 ans. Ils ont une nette préférence pour l'amusement et les vacances peu actives. Ils passent leurs vacances en France, 3 à 4 semaines au bord de la mer et une semaine aux sports d'hiver.

- Les a villages-vacances » $(25,1)$ sont âgés de 20 à 25 ans, sont actifs et parfois mariés, et vivent modestement. Ils choisissent, quand ils partent, les formules bon marché comme les villages-vacances.

- Les « aventuriers $*(21,9 \%)$, âgés de 20 à 25 ans, sont célibataires et étudiants, issus d'un milieu urbain. Adeptes des voyages improvisés, souvent à l'étranger, ils prennent des vacances longues (5 semaines) entre amis.

- Les « performants $»(16 \%)$, issus des milieux aisés, font partie de la classe d'äge des 15 a 20 ans qui vivent chez leurs parents. Ce sont les plus actifs ; ils partent plusieurs fois dans l'année, en France comme à l'étranger, pour des séjours longs (plus de 5 semaines).

- Les « guides bleus $\$(7,7 \%)$, âgés de 18 à 22 ans, sont des étudiants issus de la bourgeoisie provinciale. Traditionnels, très proches des idées de leurs parents dont ils bénéficient du soutien financier, ils sont attirés par les vacances culturelles et ne fréquentent jamais les campings.

Le panorama présenté ci-dessus tient compte des réalités sociologiques françaises. Toutefois, les jeunes expriment des aspirations marquées (enquête UNAT) pour une diversification des lieux de destination, avec un attrait pour les voyages de découverte dans des pays lointains et pour une émancipation des parents au profit des amis et d'autres groupes constitués ou non.

\section{Bibliographie}

Annuaire Statistique de la France, INSEE 2000. CREDOC (Centre de recherche pour l'étude et l'observation des conditions de vie) (1996), Consommateurs et préférences de consommation, Paris : Cahier de recherche $\mathrm{n}^{\circ} 18$.

Observatoire National du Tourisme (1998), a Le tourisme des 15 à 24 ansen France $\%$, Collection Les Essentiels du tourisme, n०10, novembre, Paris : Secrétariat d'État au Tourisme.

Rapport du Commissariat Général du Plan et de la Direction du Tourisme (1998), Rénventer les vacances, Paris : La Documentation Française.

Secrétariat d'État au Tourisme (dernière mise à jour le 20 aout 2001 ), Site Internet à consulter : http://www.tourisme.gouv.fr/STAT-CONJ/ jeunes.htm.

Trichard, Jean-François, Carole Jacquemont, et Géraldine Dreher (1997), * Le marché des 15-25 ans. De réclles imnovations... \%, Cahier Espaces $n^{0}$ 54, décembre, p. 12-19.

Union Nationale des Associations de Tourisme et de plein air (UNAT), (1994), Les vacances des jeunes français, octobre.

Village Vacances. Famille (VVF) (1996), Le tourisme des jemes. 


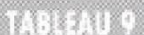

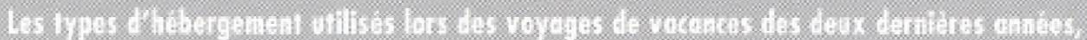

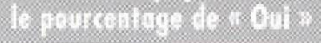

\begin{tabular}{|c|c|c|}
\hline L'HÉBERGEMENT & $\begin{array}{c}\text { Américains } \\
\frac{\gamma}{\gamma}\end{array}$ & $\begin{array}{c}\text { Canadiens } \\
\qquad \%\end{array}$ \\
\hline - Maison/appartement d'amis ou de proches & 72 & 68 \\
\hline - Hôtel/centre de villégiature/auberge rurale & 67 & 57 \\
\hline - Motel & 36 & 27 \\
\hline - Gîte touristique & 12 & 9 \\
\hline - Chalet loué & 12 & 9 \\
\hline - Chalet appartenant au répondant & 6 & 11 \\
\hline - Pavillon de chasse et péche & 5 & 2 \\
\hline $\begin{array}{l}\text { - Terrain de camping/de véhicule de } \\
\text { plaisance - service complet }\end{array}$ & 14 & 21 \\
\hline $\begin{array}{l}\text { - Terrain de camping/de véhicule de } \\
\text { plaisance - sans service }\end{array}$ & 7 & 10 \\
\hline $\begin{array}{l}\text { - Terrain de camping sans service ou } \\
\text { dans l'arriére-pays }\end{array}$ & 10 & 18 \\
\hline - Autres types de camping non spécifiés & 8 & 5 \\
\hline - Autres & 12 & 14 \\
\hline
\end{tabular}

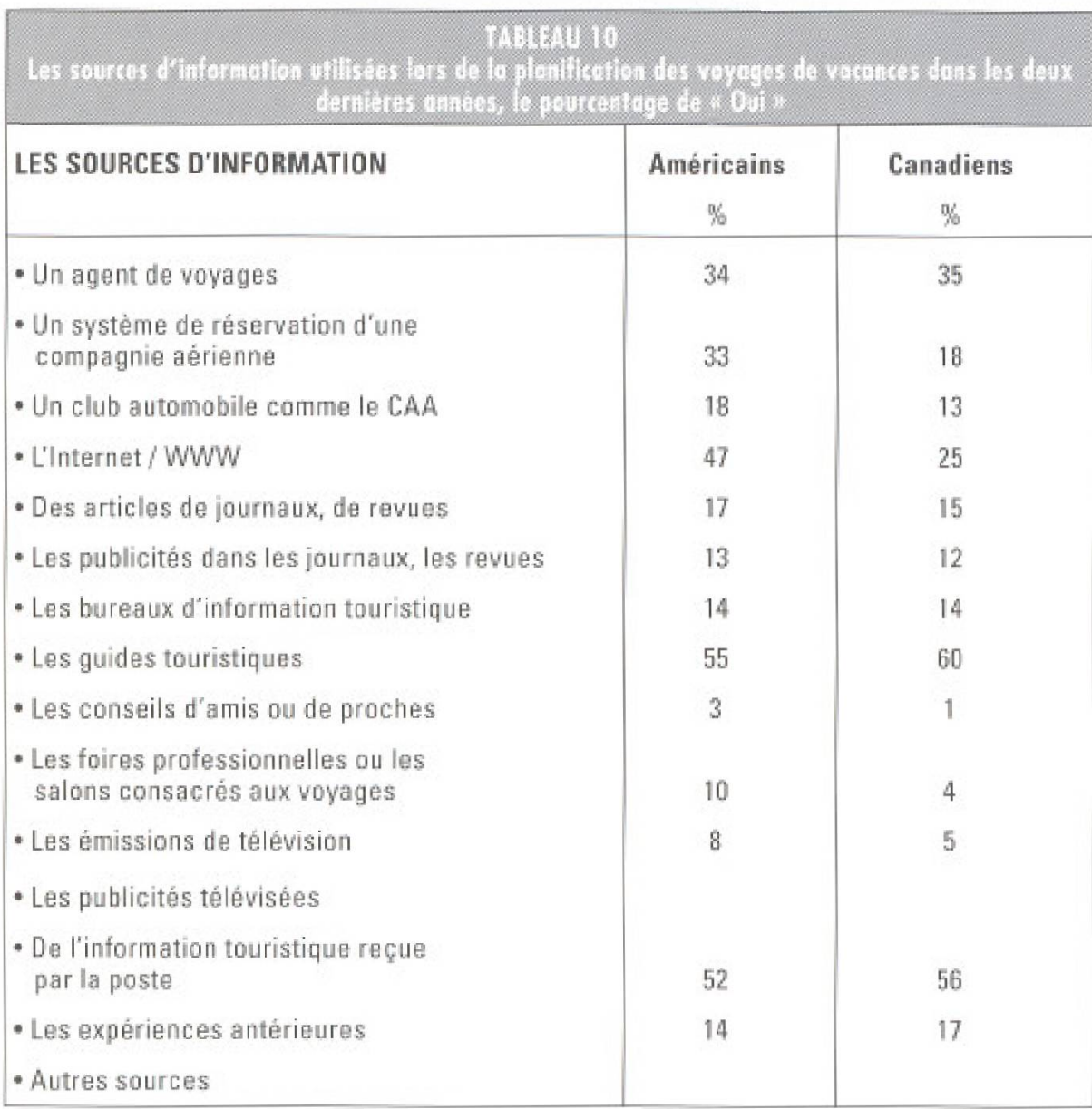

leurs expériences passétes de voyages et sur l'utilisation de l'Internet.

Les principaux objectifs des voyages de vacances évoqués par les jeunes sont : se reposer et se détendre, passer du temps en famille, visiter des amis et être à la recherche d'une « aventure excitante $\%$.

\section{Les similitudes des comportements touristiques des jeunes Américains et des jeunes Canadiens}

Les similitudes des comportements touristiques des jeunes Américains et des jeunes Canadiens sont très fortes. On peut parler d'un marché unique où les convergences culturelles (au sens ethnologique) sont trè̀s fortes; ces jeunes parlent la même langue et consomment les mêmes produits économiques (biens de consommation) et les mêmes produits culturels (musique, cinéma, télévision, lecture, etc.).

En ce qui concerne les comportements touristiques, pour la plupart des variables considérées dans cet article, la corrélation entre les jeunes Américains et les jeunes Canadiens est très élevée. Dans $73 \%$ des variables comparées, où la mesure de la corrélation a été possible, les coefficients de corrélation (Bravais-Pearson) dépassent 0,90 . Les variables où les corrélations sont les plus faibles (des plus élevées aux plus faibles corrélations) entre les deux groupes de jeunes sont:

- les destinations de vacances (tableau 3):

- le nombre de semaines de vacances (tableau 1).

L'Amérique du Nord, en tout cas celle des jeunes, devient de plus en plus homogène en ce qui concerne les comportements de vacances.

\section{Note}

I Il s'agit de voyages de vacances de quatre nuitées ou plus hors du domicile habituel, effectués dans les deux demières années, principalement pour le plaisir, y compris pour visiter des amis ou des proches (Statistique Canada). 